\title{
Observation of $\mathcal{P} \mathcal{T}$ phase transition in a simple mechanical system
}

\author{
Carl M. Bender ${ }^{1 *}$, Bjorn K. Berntson ${ }^{2 \dagger}$, David Parker ${ }^{1 \ddagger}$, and E. Samuel ${ }^{1 \S}$ \\ ${ }^{1}$ Department of Physics, King's College London, Strand, London WC2R 1LS, UK \\ ${ }^{2}$ Blackett Laboratory, Imperial College, London $S W^{\text {r }}$ 2AZ, UK
}

(Dated: June 22, 2012)

\begin{abstract}
If a Hamiltonian is $\mathcal{P} \mathcal{T}$ symmetric, there are two possibilities: Either the eigenvalues are entirely

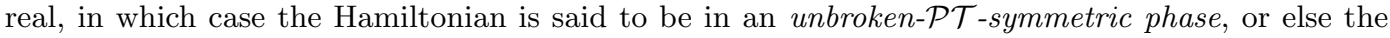
eigenvalues are partly real and partly complex, in which case the Hamiltonian is said to be in a

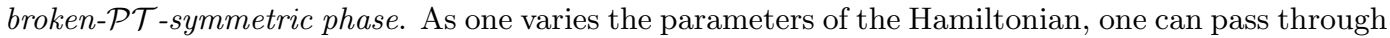
the phase transition that separates the unbroken and broken phases. This transition has recently been observed in a variety of laboratory experiments. This paper explains the phase transition in a simple and intuitive fashion and then describes an extremely elementary experiment in which the phase transition is easily observed.
\end{abstract}

PACS numbers: 11.30.Er, 03.65.Ca, 03.65.Xp

\section{INTRODUCTION}

Quantum-mechanical $\mathcal{P} \mathcal{T}$-symmetric Hamiltonians often exhibit two parametric regions, a region of unbroken $\mathcal{P} \mathcal{T}$ symmetry in which the eigenvalues are all real, and a region of broken $\mathcal{P} \mathcal{T}$ symmetry in which some of the eigenvalues are real and the remaining eigenvalues are complex. (Here, $\mathcal{P}$ represents space reflection, or parity, and $\mathcal{T}$ represents time reversal.) A phase transition occurs at the boundary between these two regions. This paper describes an elementary experiment that demonstrates the $\mathcal{P} \mathcal{T}$ phase transition in a simple $\mathcal{P} \mathcal{T}$-symmetric classical-mechanical system involving two coupled driven pendula.

If a quantum-mechanical Hamiltonian is Dirac Hermitian (that is, if $H=H^{\dagger}$, where $H^{\dagger}$ is the transpose and complex conjugate of $H$ ), then its energy eigenvalues are always real. Thus, a Dirac Hermitian Hamiltonian can never exhibit a phase transition where its eigenvalues go from being real to being complex. However, in 1998 it was shown that the eigenvalues of a $\mathcal{P} \mathcal{T}$-symmetric Hamiltonian can be entirely real, even if the Hamiltonian is not Dirac Hermitian [1 3]. The class of $\mathcal{P} \mathcal{T}$-symmetric Hamiltonians considered in Refs. [1-3] has the form

$$
H=p^{2}+x^{2}(i x)^{\varepsilon},
$$

where $\varepsilon$ is a real parameter. These Hamiltonians are not Dirac Hermitian (except at $\varepsilon=0$ ), but they are $\mathcal{P} \mathcal{T}$ symmetric because under space reflection $x$ changes sign and under time reversal $i$ changes sign. The Hamiltonians (1) exhibit a region of unbroken $\mathcal{P} \mathcal{T}$ symmetry $(\varepsilon \geq 0)$ and a

\footnotetext{
*Permanent address: Department of Physics, Washington University, St. Louis, MO 63130, USA.

email: cmb@wustl.edu

†email: bjorn.berntson11@imperial.ac.uk

‡email: david.j.parker@kcl.ac.uk

§email: ernest.samuel@kcl.ac.uk
}

region of broken symmetry $(-1<\varepsilon<0)$. Thus, at $\varepsilon=1$ and at $\varepsilon=2$ we obtain the Hamiltonians

$$
H=p^{2}+i x^{3}
$$

and

$$
H=p^{2}-x^{4},
$$

and surprisingly, the eigenvalues of these Hamiltonians are all real, positive, and discrete. However, at $\varepsilon=-1 / 2$ we obtain the Hamiltonian $H=p^{2}+x^{2}(i x)^{-1 / 2}$, which has an infinite number of complex eigenvalues and only a finite number of real eigenvalues.

Recently, the $\mathcal{P} \mathcal{T}$ phase transition has been observed in laboratory experiments on many different $\mathcal{P} \mathcal{T}$-symmetric systems [4-12]. The coupled-electronic-oscillator experiment by J. Schindler et al. [11] is particularly elegant because of its simplicity, and we were motivated by this experiment to construct its mechanical analog by using a pair of coupled driven pendula. We have been able to do this, and in this mechanical system the $\mathcal{P} \mathcal{T}$ phase transition is easy to observe.

Mechanical oscillators (coupled pendula) have been used in other contexts to model complex physical phenomena. For example, in particle physics, it was proposed that transitions in $\mathrm{K}^{0} \overline{\mathrm{K}}^{0}, \mathrm{~B}^{0} \overline{\mathrm{B}}^{0}$, and $\mathrm{D}^{0} \overline{\mathrm{D}}^{0}$ systems could be visualized by using coupled pendula 13 . These experiments have recently been performed [14, 15. In these experiments, systems of coupled, damped pendula were used. The experiment reported in this paper differs from these experiments in that while one of our pendula is damped, the other is undamped (driven).

This paper is organized as follows. Section II presents an intuitive explanation of the $\mathcal{P} \mathcal{T}$ phase transition. The pendulum experiment is described in Sec. III Section IV gives concluding remarks and proposes some follow-up experiments that we believe can be readily performed. 


\section{INTUITIVE EXPLANATION OF THE $\mathcal{P} \mathcal{T}$ PHASE TRANSITION}

Originally, $\mathcal{P} \mathcal{T}$-symmetric quantum mechanics was studied at a highly mathematical level. In Ref. [1, the techniques of complex variables, asymptotics, differential equations, and perturbative theory were used to understand the analytic continuation of eigenvalue problems and $\mathcal{P} \mathcal{T}$ symmetry. The rigorous proof that the eigenvalues of $H$ in (1) are real and positive when $\varepsilon \geq 0$ involves the use of sophisticated mathematical techniques (Baxter TQ relation, Bethe ansatz, functional determinants), which are used to study integrable systems and conformal field theory [16, 17.

However, as experiments on $\mathcal{P} \mathcal{T}$-symmetric physical systems have been performed and published, we have realized that the $\mathcal{P} \mathcal{T}$ phase transition can be explained simply and intuitively without resorting to sophisticated mathematics. We present this explanation below.

Imagine two identical closed and isolated boxes, one on the negative- $x$ axis at $x=-a$, and the other on the positive- $x$ axis at $x=a$ (see Fig. 1). Inside the left box is a sink (an antenna that absorbs energy) and inside the right box is a source (an antenna that radiates energy at an equal rate). This system is $\mathcal{P} \mathcal{T}$ symmetric because under parity $\mathcal{P}$ the left box and the right box interchange positions and under time reversal $\mathcal{T}$ the sink becomes a source and the source becomes a sink.
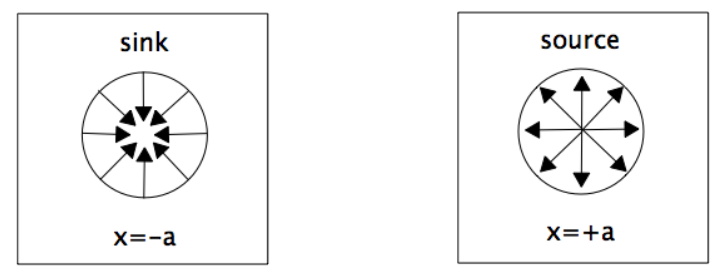

FIG. 1: A simple schematic $\mathcal{P} \mathcal{T}$-symmetric physical system: A box located at $x=-a$ contains a sink (an antenna that absorbs) and a box located at $x=+a$ contains a source (an antenna that radiates at an equal rate). Under space reflection $\mathcal{P}$ the boxes interchange, and under time reversal $\mathcal{T}$ the sink becomes a source and the source becomes a sink. Thus, the system is $\mathcal{P} \mathcal{T}$ symmetric. If the boxes are isolated, the system cannot be in equilibrium because the energy decays to zero in the left box and grows to infinity in the right box. Thus, in this case the $\mathcal{P} \mathcal{T}$ symmetry of the system is broken. The energies corresponding to these runaway modes are complex. However, if the boxes are sufficiently strongly coupled, the system can equilibrate and the $\mathcal{P} \mathcal{T}$ symmetry is unbroken. When the system is in equilibrium, its energy is real.

The Hamiltonian that describes the time evolution of the one-dimensional system in the left box is the $1 \times 1$ matrix $H=\left[E_{1}\right]=\left[a e^{i \theta}\right]$, where $a>0$ and $0<\theta<\pi$ so that $\operatorname{Im} E_{1}>0$. The solution to the time-dependent
Schrödinger equation for this system,

$$
-i \frac{d}{d t} \phi(t)=H \phi(t)
$$

is $\psi(t)=\psi(0) e^{i E_{1} t}$, which decays exponentially with time because there is a sink in the left box.

Similarly, the Hamiltonian that describes the time evolution of the one-dimensional system in the right box is the $1 \times 1$ matrix $H=\left[E_{2}\right]=\left[a e^{-i \theta}\right]$, so that $\operatorname{Im} E_{2}<0$. The solution to the time-dependent Schrödinger equation for the right system is $\psi(t)=\psi(0) e^{i E_{2} t}$, which grows exponentially with time because there is a source in the right box.

The left and right systems taken together are described by the $2 \times 2$ diagonal matrix Hamiltonian

$$
H=\left[\begin{array}{cc}
a e^{i \theta} & 0 \\
0 & a e^{-i \theta}
\end{array}\right]
$$

This Hamiltonian is not Dirac Hermitian and its eigenvalues $E_{1}$ and $E_{2}=E_{1}^{*}$ are complex. However, it is $\mathcal{P} \mathcal{T}$ symmetric, where the parity operator $\mathcal{P}$ is the matrix

$$
\mathcal{P}=\left[\begin{array}{ll}
0 & 1 \\
1 & 0
\end{array}\right],
$$

which interchanges the two systems, and the time reversal operator $\mathcal{T}$ is complex conjugation. The system described by $H$ in (5) is not in equilibrium because the eigenfunction in the left box decays exponentially and the eigenfunction in the right box grows exponentially. Correspondingly, $E_{1}$ and $E_{2}$, the eigenvalues of $H$, are complex. Thus, the system is in a broken- $\mathcal{P} \mathcal{T}$-symmetric phase.

The combined two-box system can achieve equilibrium if we couple the boxes together sufficiently strongly. We couple the boxes symmetrically in order to preserve the $\mathcal{P} \mathcal{T}$ symmetry of the entire system:

$$
H=\left[\begin{array}{cc}
a e^{i \theta} & g \\
g & a e^{-i \theta}
\end{array}\right],
$$

where the coupling constant $g$ is real. This coupling allows the excess energy in the right box to leak into the left box.

The eigenvalues of $H$ in (7) become real if $g^{2}>$ $a^{2} \sin ^{2} \theta$. Thus, if the coupling is strong enough, the excess energy in the left box can flow into the right box fast enough for the system to remain in equilibrium. When this happens, the energy eigenvalues are real and the system is in an unbroken- $\mathcal{P} \mathcal{T}$-symmetric phase. The phase transition occurs when the coupling constant $g$ exceeds the critical value given by

$$
g_{\text {crit }}^{2}=a^{2} \sin ^{2} \theta
$$

Using this simple two-dimensional model, we can now understand heuristically why the Hamiltonians in (1) 
have an unbroken $\mathcal{P} \mathcal{T}$ symmetry when $\varepsilon>0$ and a broken $\mathcal{P} \mathcal{T}$ symmetry when $\varepsilon<0$. Consider, for example, the potential $i x^{3}$ for $H$ in (2); this potential has a positive-imaginary part when $x>0$ and a negativeimaginary part when $x<0$. Thus, there is a nonlocal source everywhere on the negative- $x$ axis and a corresponding nonlocal sink everywhere on the positive- $x$ axis. Can such a system actually be in equilibrium? One might think that it would be impossible for this system to reach equilibrium because the source becomes infinitely strong as $x \rightarrow-\infty$ and the sink becomes infinitely strong as $x \rightarrow+\infty$. However, by means of a simple classical argument, we can easily show that the system can indeed achieve equilibrium: We determine how long it takes for particles at $x=-\infty$ to flow to $x=+\infty$. The classical time of flight $T$ is given by

$$
T=\int d t=\int \frac{d x}{p}=\int_{x=-\infty}^{\infty} \frac{d x}{\sqrt{E-i x^{3}}},
$$

where we have used the condition that the Hamiltonian represents the classical energy: $E=p^{2}+i x^{3}$. Since the integral in (9) converges, the time of flight from $x=-\infty$ to $x=+\infty$ is finite, and thus the system can attain equilibrium. Clearly, if $\varepsilon$ in (1) becomes negative, the time of flight integral diverges and the system cannot be in equilibrium. This is why the $\mathcal{P} \mathcal{T}$ symmetry of $H$ breaks and the eigenvalues become complex when $\varepsilon<0$.

This simple two-box source-and-sink model explains what was done in the microwave cavity experiment in Ref. [12]. In this experiment two coupled microwave cavities, one containing a source of microwaves and the other containing a sink of microwaves was used, and as the coupling was varied, the $\mathcal{P} \mathcal{T}$ phase transition was observed.

The source-and-sink model also explains the twochannel optics experiments in Refs. [6] and [7. In these experiments light travels down a coupled pair of wave guides. One wave guide has loss and the other has gain. If the coupling of the wave guides is sufficiently strong, then the system is in equilibrium, and one observes Rabi oscillations (power oscillations) in which the optical energy oscillates between the two wave guides. When the coupling between the wave guides becomes too weak, the Rabi oscillations cease and the system can no longer remain in equilibrium; the power grows exponentially in one wave guide and decays exponentially in the other.

The experiment in Ref. [11] is the electronic analog of the two-channel optics experiments. The electronic experiment involves two inductively-coupled LRC circuits, one with gain and the other with loss. Once again, as the coupling between the two oscillators becomes weaker than a critical value, the system enters the $\mathcal{P} \mathcal{T}$-broken phase and is no longer in equilibrium.

\section{DESCRIPTION OF THE EXPERIMENT}

The experiment discussed in this paper is a classicalmechanical analog of the electronic experiment in
Ref. 11. Instead of two LRC circuits, there are two coupled pendula, one with energy loss and one with energy gain. We first formulate some elementary equations to model a coupled a two-pendulum system and then we describe the experiment itself.

\section{A. An overly simple mathematical model of a two-oscillator system}

The Hamiltonian

$$
H=\frac{1}{2} p^{2}+\frac{1}{2} x^{2}+\frac{1}{2} q^{2}+\frac{1}{2} y^{2}+\varepsilon x y
$$

describes the small-amplitude frictionless motion of two coupled pendula, where $x(t)$ and $y(t)$ are the displacements of the pendula. The parameter $\varepsilon$ represents the coupling of the two pendula. The classical equations of motion for $x(t)$ and $y(t)$ are

$$
\begin{aligned}
& x^{\prime \prime}(t)=-x(t)-\varepsilon y(t), \\
& y^{\prime \prime}(t)=-y(t)-\varepsilon x(t) .
\end{aligned}
$$

If we solve these equations numerically, we observe Rabi oscillations (see Fig. 2).

A simple, but as we will see not a good, way to create a $\mathcal{P} \mathcal{T}$-symmetric system is to introduce a damping (loss) term in the $x$ equation and an undamping (gain) term in the $y$ equation:

$$
\begin{aligned}
& x^{\prime \prime}(t)+a x^{\prime}(t)+x(t)+\varepsilon y(t)=0, \\
& y^{\prime \prime}(t)-a y^{\prime}(t)+y(t)+\varepsilon x(t)=0,
\end{aligned}
$$

where the damping/undamping parameter $a$ is positive. This system is $\mathcal{P} \mathcal{T}$ symmetric because $x$ and $y$ are interchanged under $\mathcal{P}$ and the signs of the damping and undamping terms reverse under time reversal $\mathcal{T}$.

To solve 12 analytically we rewrite these equations as a system of four first-order constant-coefficient differential equations:

$$
\begin{aligned}
x^{\prime}(t) & =p(t), \\
p^{\prime}(t) & =-x(t)-\varepsilon y(t)-a p(t), \\
y^{\prime}(t) & =q(t), \\
q^{\prime}(t) & =-y(t)-\varepsilon x(t)+a q(t) .
\end{aligned}
$$

We then express these differential equations compactly in matrix form:

$$
\mathbf{V}^{\prime}(t)=\mathbf{M V}(t),
$$

where the matrix $\mathbf{M}$ and the vector $\mathbf{V}$ are

$$
M=\left[\begin{array}{cccc}
0 & 1 & 0 & 0 \\
-1 & -a & -\varepsilon & 0 \\
0 & 0 & 0 & 1 \\
-\varepsilon & 0 & -1 & a
\end{array}\right] \text { and } \mathbf{V}=\left[\begin{array}{c}
x(t) \\
p(t) \\
y(t) \\
q(t)
\end{array}\right]
$$



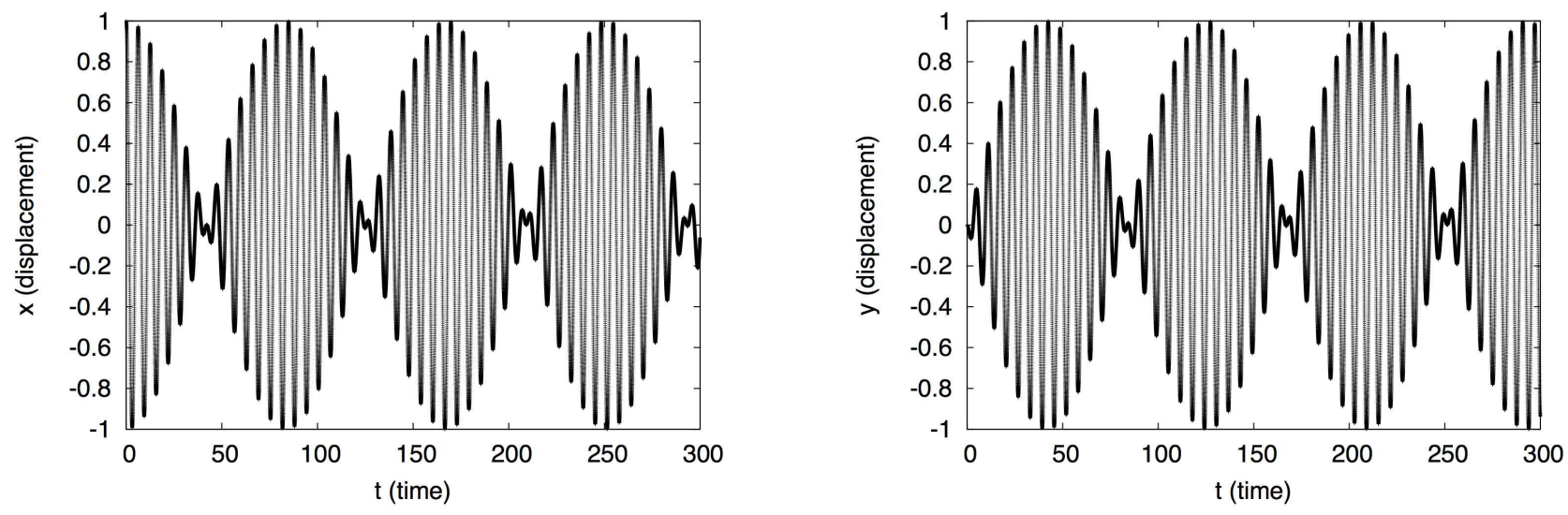

FIG. 2: Oscillatory motion of a pair of coupled pendula [see [11] ] with no loss or gain. The $x$ displacement is shown in the left panel and the $y$ displacement is shown in the right panel. The coupling parameter is $\varepsilon=0.075$. Rabi power oscillations occur in which the maximum and minimum displacements $x(t)$ and $y(t)$ are $90^{\circ}$ out of phase.

To solve 15 we must find the eigenvalues $E$ of the matrix $M$. To do so we calculate the determinant $D$ of the matrix $\mathbf{M}-E \mathbf{I}$ :

$$
D=\operatorname{det}\left[\begin{array}{cccc}
-E & 1 & 0 & 0 \\
-1 & -a-E & -\varepsilon & 0 \\
0 & 0 & -E & 1 \\
-\varepsilon & 0 & -1 & a-E
\end{array}\right]
$$

The determinant $D$ is a simple quadratic form in $E^{2}$,

$$
D=E^{4}+\left(2-a^{2}\right) E^{2}+1-\varepsilon^{2} .
$$

The solution to the equation $D=0$ is

$$
E^{2}=\frac{1}{2}\left(a^{2}-2 \pm \sqrt{a^{4}-4 a^{2}+4 \varepsilon^{2}}\right) .
$$

The system 13 exhibits oscillatory behavior if $E^{2}<$ 0 . Two conditions must be met for $E^{2}$ to be negative:

$$
\begin{aligned}
& a^{4}-4 a^{2}+4 \varepsilon^{2}>0, \\
& a^{2}-2+\sqrt{a^{4}-4 a^{2}+4 \varepsilon^{2}}<0 .
\end{aligned}
$$

Condition (2) immediately gives $\varepsilon<1$. Then, Condition (1) implies that

$$
a<a_{\mathrm{crit}}=2\left(1-\sqrt{1-\varepsilon^{2}}\right) .
$$

Therefore, if the damping/undamping parameter $a$ is below this critical value, the eigenvalues are imaginary and the system is in the unbroken- $\mathcal{P} \mathcal{T}$-symmetric region.

As the damping/undamping parameter $a$ increases from 0 , the frequency of the Rabi power oscillations increases. Eventually, at the critical critical value of $a$ the Rabi frequency is the same as the oscillation frequency.
Above this value of $a$ we enter the region of broken $\mathcal{P} \mathcal{T}$ symmetry; the Rabi oscillations cease and we observe the onset of exponential behavior. However, a numerical plot reveals the shortcomings of the overly simple model (12). In Fig. 3 we plot the $x(t)$ and $y(t)$ for the case $a=\varepsilon=0.075$. Observe that $y(t)$ grows exponentially, but after an initial period of exponential decay $x(t)$ also grows exponentially. This is because a balanced damping and undamping violates energy conservation. For the model equations (12), the rate of growth of $y(t)$ due to undamping is proportional to the velocity of $y(t)$ and the rate of decay of $x(t)$ is proportional to the velocity of $x(t)$. Thus, as $y(t)$ gets big and $x(t)$ gets small, the loss of energy in the $x$ oscillator becomes insignificant while the growth of energy in the $y$ oscillator becomes immense. Then, because the two oscillators are coupled, $x(t)$ eventually becomes large because the energy in the $y$ oscillator leaks into the $x$ oscillator.

\section{B. An improved energy-conserving oscillator model}

Evidently, we need to construct a better model in which the source antenna radiates the same amount of energy as is absorbed by the sink antenna. We do this by constructing a pair of difference-differential equations: We begin with the coupled-oscillator equations in (11) in which there is no loss or gain. We then impose the additional condition that whenever $x(t)$ reaches an amplitude maximum, we remove a given fixed fraction of the energy $g$ from the $x$ oscillator (by decreasing the amplitude appropriately and reinitializing the motion). Then, when $y(t)$ is next at a maximum we transfer this exact amount of energy to the $y$ oscillator at its peak by increasing its amplitude accordingly. 

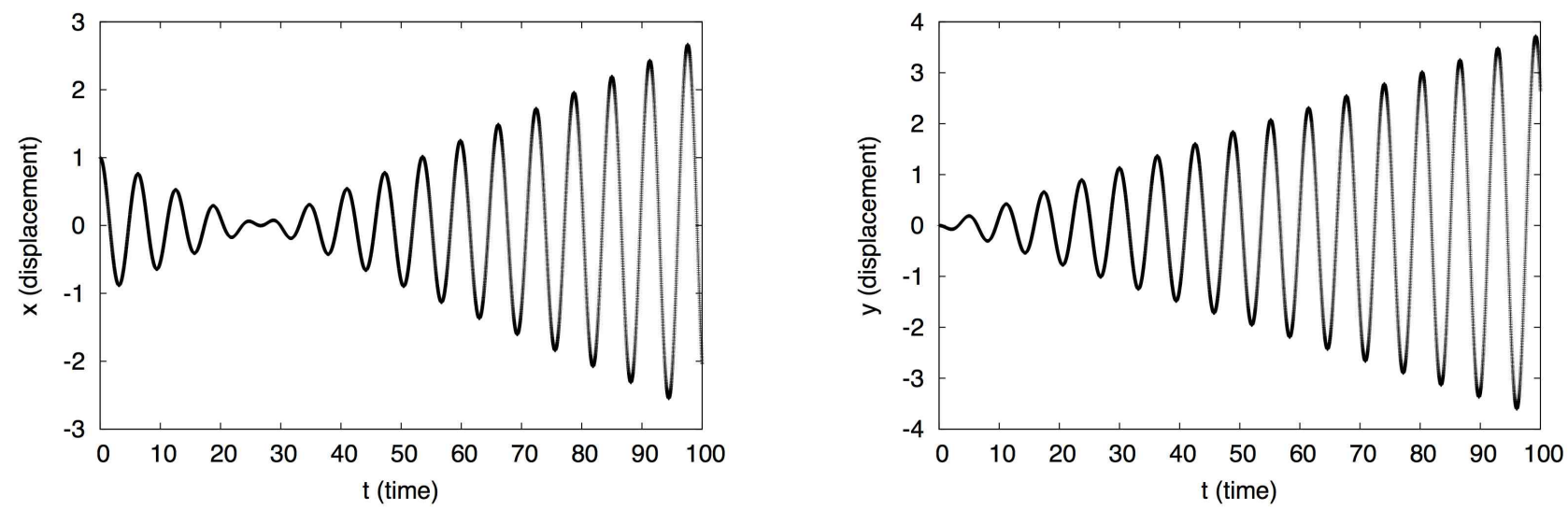

FIG. 3: Numerical solution to the overly simple coupled-oscillator equations 12 for the parameter choice $a=\varepsilon=0.075$, which is deep in the broken- $\mathcal{P} \mathcal{T}$-symmetric region. The $x$ displacement is shown in the left panel and the $y$ displacement is shown in the right panel. As expected, the $y$ oscillator exhibits gain, but after an initial period of decay, the $x$ oscillator also exhibits gain. This is because the system does not conserve energy, and the excess energy in the $y$ oscillator leaks into the $x$ oscillator.

Numerical solutions for this improved oscillator model are shown in Figs. 4 and 5 . In Fig. 4 we show what happens if we take $\varepsilon=0.05$ and take $g=0.01$. Here, we remove $1 \%$ of the energy of the $x$ oscillator each time it reaches its maximum amplitude and then transfer this energy to the $y$ oscillator when it reaches its next amplitude maximum; this transfer reduces the amplitude of the $x$ oscillator at its maximum to $\sqrt{0.99}=99.5 \%$ of its former value. The coupling $\varepsilon=0.05$ is large enough to keep the system in the region of unbroken $\mathcal{P} \mathcal{T}$ symmetry. The signal that the $\mathcal{P} \mathcal{T}$ symmetry is not broken is that the Rabi oscillations persist and the amplitudes of the oscillators remain constant.

In Fig. 5 we take the coupling smaller $\varepsilon=0.01$ and $g$ larger $g=0.3$. [Now, at each swing only $70 \%$ of the energy in the $x$ oscillator remains; when we make this transfer we reduce the peak amplitude to $\sqrt{0.7}=83.7 \%$ of its former value. These amplitude reductions are clearly visible in the graph of $x(t)$.] In this case the energy transfer overwhelms the small coupling of the oscillators and we are in the broken- $\mathcal{P} \mathcal{T}$-symmetric region. The signal that the $\mathcal{P} \mathcal{T}$ symmetry is broken is that the Rabi oscillations cease and the amplitudes of the $x$ (and $y$ ) oscillators decrease (and increase) towards their limiting values.

\section{Description of the experimental setup}

The experiment approximates the energy-conserving mathematical oscillator model very closely. Two identical pendula are suspended from a horizontal rope of total length $94 \mathrm{~cm}$ (see Fig. 6). By adjusting the tension in the horizontal rope, we can vary the coupling of the two pendula. Each pendulum consists of a 50-gram cylindri- cal mass (diameter $2.3 \mathrm{~cm}$, height $4.4 \mathrm{~cm}$ ) hanging from a string of length $39 \mathrm{~cm}$. The separation of the two pendula is $34 \mathrm{~cm}$.

In order to add or subtract energy from a pendulum, an electromagnet is situated about $5 \mathrm{~cm}$ from top of each string. When the electromagnet is turned on, it applies a horizontal force to a small iron nail that is attached to the string supporting the bob. The electromagnets are only on for about $10 \mathrm{msec}$, so that they provide a brief impulse to the string. The impulse is applied to the left pendulum when the pendulum is moving away from the electromagnet, so the effect of the impulse is to subtract a small amount of kinetic energy from the pendulum. However, the impulse is applied to the right pendulum when the pendulum is moving towards the electromagnet, so the effect of the impulse is to add a small amount of kinetic energy to the pendulum.

Each of the electromagnets is triggered by a pair of optical sensors located just above the pendulum bobs. For the electromagnet to fire, the two optical sensors must be triggered in a prescribed order, so the output of the optical sensors is fed into a simple logic circuit.

To record the motion of the pendula we use a small video camera mounted on a goose-neck stand [18. The camera looks up from underneath each pendulum and records the instantaneous position of the pendulum 15 times per second. We then draw a curve through these data points to display the motion of the pendulum.

We begin our experiment by taking data with the magnets turned off (see Fig. 7). The tension in the horizontal rope is 200 grams. The key characteristic feature of the swinging pendula is that they exhibit Rabi power oscillations. The experimental data are in qualitative agreement with the theoretical predictions in Fig. 2, [Note 

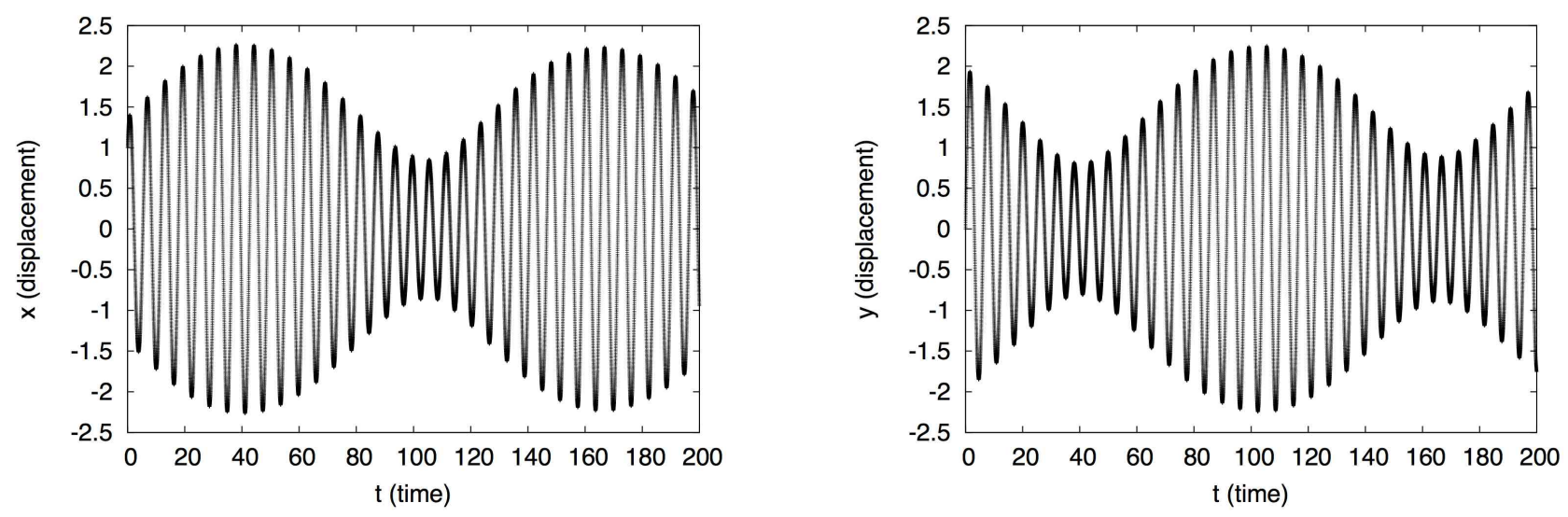

FIG. 4: Mathematical simulation of the improved energy-conserving oscillator model in the unbroken- $\mathcal{P} \mathcal{T}$ region. In this graph $\varepsilon=0.05$ and $g=0.01$. (That is, $1 \%$ of the energy in the $x$ oscillator is removed each time $x$ reaches a peak, and this exact amount of energy is then transferred to the $y$ oscillator when it reaches a peak. This transfer reduces the peak- $x$ amplitude to $99.5 \%$ of its former value, which is too small to be seen on this graph.) The motion of the $x$ oscillator is shown in the left panel and the motion of the $y$ oscillator is shown in the right panel. We can see that the $\mathcal{P} \mathcal{T}$ symmetry is not broken: The Rabi oscillations persist and the amplitudes of the oscillators remain constant.

that the simple theoretical model $(12)$ with vanishing damping/undamping parameter $a$ is the same as the im- proved energy-conserving oscillator model with vanishing energy-transfer parameter $g$.
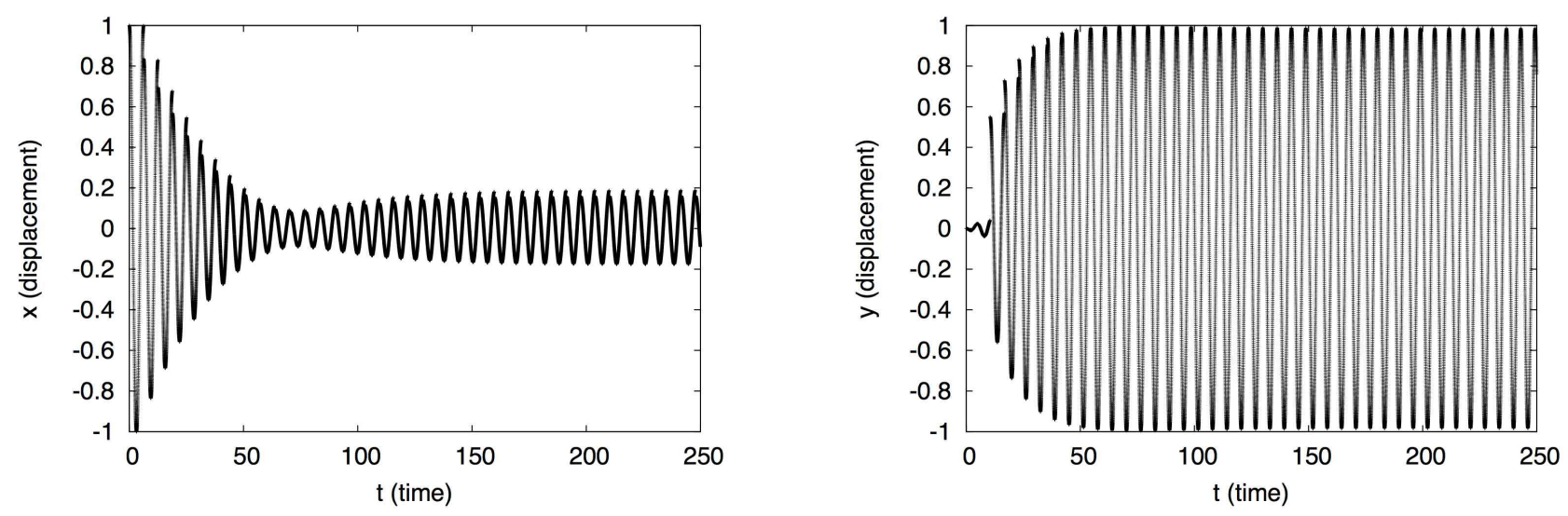

FIG. 5: Mathematical simulation of the improved energy-conserving oscillator model in the broken- $\mathcal{P} \mathcal{T}$ region. The coupling here is $\varepsilon=0.01$, which is smaller than that in Fig. 4 Also, $g=0.3$, which is larger than that in Fig. 4 . For this value of $g$, 30\% of the energy in the $x$ oscillator is removed each time $x$ reaches a peak, and this exact amount of energy is then transferred to the $y$ oscillator when it reaches a peak. This transfer reduces the peak amplitude to 0.837 of previous value, and this change can be seen in the plot of $x(t)$. The motion of the $x$ oscillator is shown in the left panel and the motion of the $y$ oscillator is shown in the right panel. Observe that the Rabi oscillations cease and that the $x$ oscillations die down to a limiting amplitude and correspondingly the $y$ oscillations increase to a limiting amplitude. This is the characteristic behavior of an oscillator system having a broken $\mathcal{P} \mathcal{T}$ symmetry.

Next, we turn on the magnets weakly and increase the coupling of the pendula by increasing the tension 


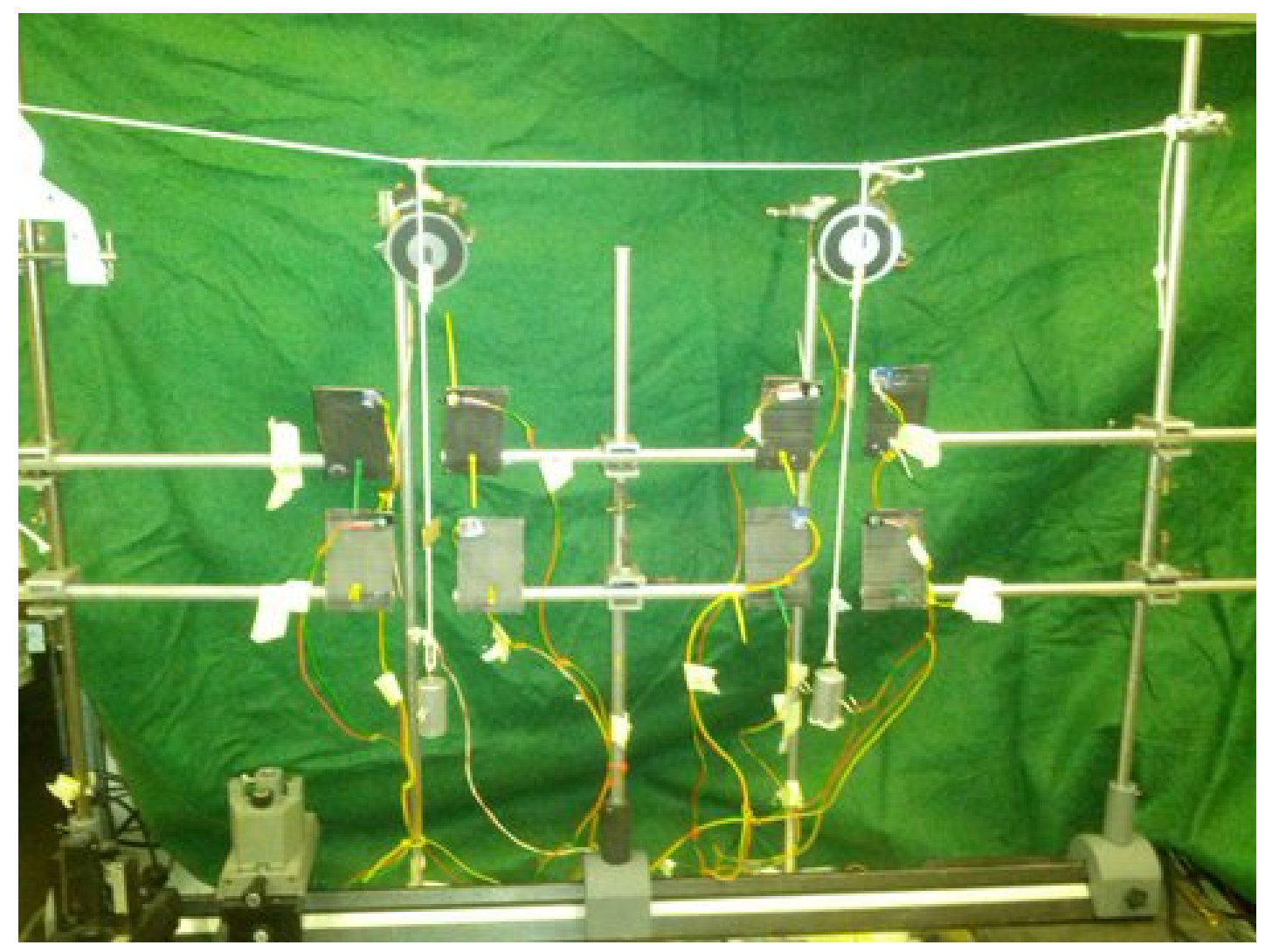

FIG. 6: View of the two-pendulum experiment. Two pendula are suspended from a horizontal rope and the tension in the rope is adjusted to increase or decrease the coupling of the pendula. The horizontal rope runs around a wheel to the left (not shown) and is attached to a tray upon which weights can be added or subtracted to change the tension. Electromagnets near the top of the strings supporting the bobs apply brief impulses to small iron nails attached by white tape to the strings. The electromagnets are triggered by pairs of optical sensors just above the pendulum bobs. The electromagnets are timed so that on each swing a small amount of kinetic energy is subtracted from the left pendulum and a roughly equal amount of kinetic energy is added to the right pendulum.

in the string from which the pendula hang from 200 to 400 grams. The experimental data are shown in Fig. 8. Note that the Rabi power oscillations persist. Again, the experimental data are in qualitative agreement with the theoretical predictions in Fig. 4. We conclude that we are still in the region of unbroken $\mathcal{P} \mathcal{T}$ symmetry.

Finally, we make the magnets stronger and increase the tension in the string from 400 grams to 600 grams in order to weaken the coupling of the pendula. The experimental data is shown in Fig. 9. If we compare this data to the theoretical predictions in Fig. 5 , we again observe good qualitative agreement: The Rabi power oscillations have ceased and we can see that the amplitudes of the pendula level off and rapidly approach their asymptotic values. We conclude that we have entered the region of broken $\mathcal{P} \mathcal{T}$ symmetry.

\section{FINAL REMARKS}

In summary, the phase transition between an unbroken and a broken $\mathcal{P} \mathcal{T}$-symmetric phase is easy to explain at an intuitive level. It is simply a matter of whether two systems, one with gain and the other with loss, are coupled strongly enough to be in equilibrium. The phase transition, which takes place at a critical value of the coupling, can be observed in a mechanical system consisting of two coupled pendula, one with damping and the other with undamping. In the unbroken phase we observe periodic Rabi power oscillations. However, when the damping/undamping becomes strong relative to the strength of the coupling, the system enters a broken- $\mathcal{P} \mathcal{T}$ symmetric phase. At this transition the Rabi power oscillations cease, and the amplitudes of the pendula approach limiting values.

A natural extension of the experimental work described in this paper to investigate $\mathcal{P} \mathcal{T}$-symmetric chains of coupled pendula with alternating damping and undamping. It is predicted that an optical system consist- 

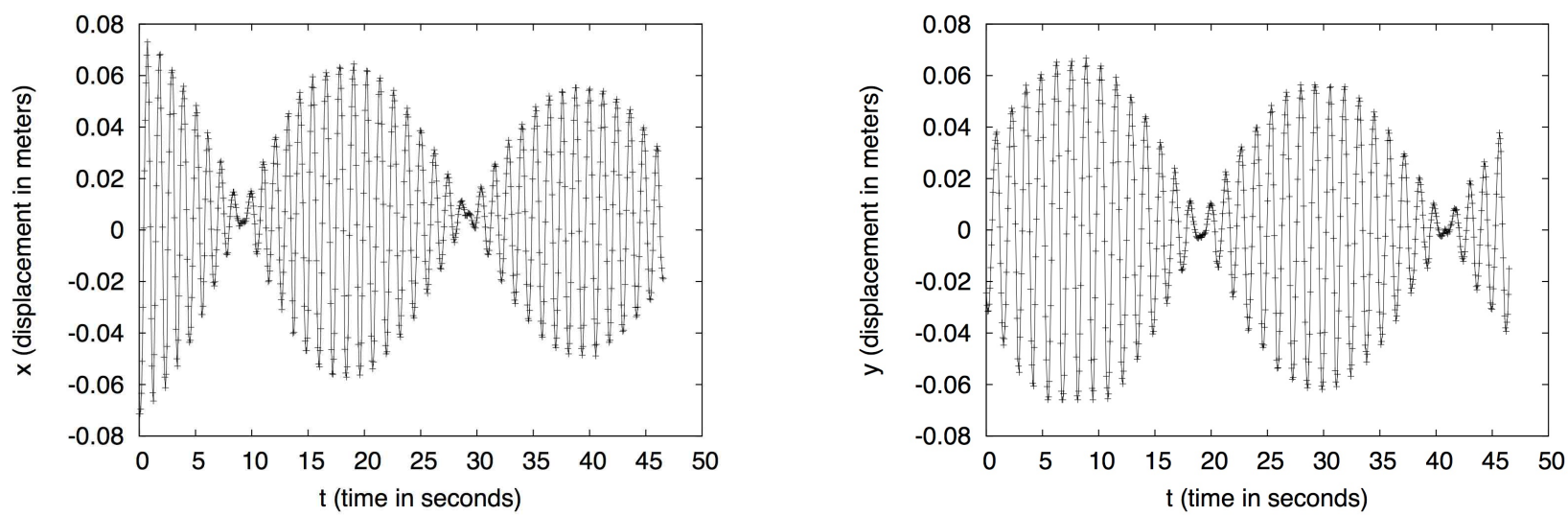

FIG. 7: Experimentally measured motion of the pendula with the magnets turned off. The tension in the string is 200 grams. To produce these graphs we use a camera that records the instantaneous position of each pendulum (tick marks on the graph) 15 times per second. We then fit a curve through these data points. The motion of the pendula is qualitatively similar to that in the theoretical curves in Fig. 2. In this configuration the $\mathcal{P} \mathcal{T}$ symmetry is unbroken. The signal for an unbroken $\mathcal{P} \mathcal{T}$ symmetry is the presence of Rabi power oscillations, which are $90^{\circ}$ out of phase. One can observe a slight decay in the amplitudes of the pendula due to friction.

ing of coupled optical fibers with alternating loss and gain will exhibit birefringence [19]. Our objective in studying this multiple pendulum system is to observe the mechanical equivalent of birefringence.

We have also begun to study $\mathcal{P} \mathcal{T}$-symmetric systems of coupled accoustic wave guides. We have already found that coupled accoustic wave guides with alternating loss and gain can exhibit a rich and elaborate array of phase transitions.

CMB thanks the U.K. Leverhulme Foundation and the U.S. Department of Energy for financial support.
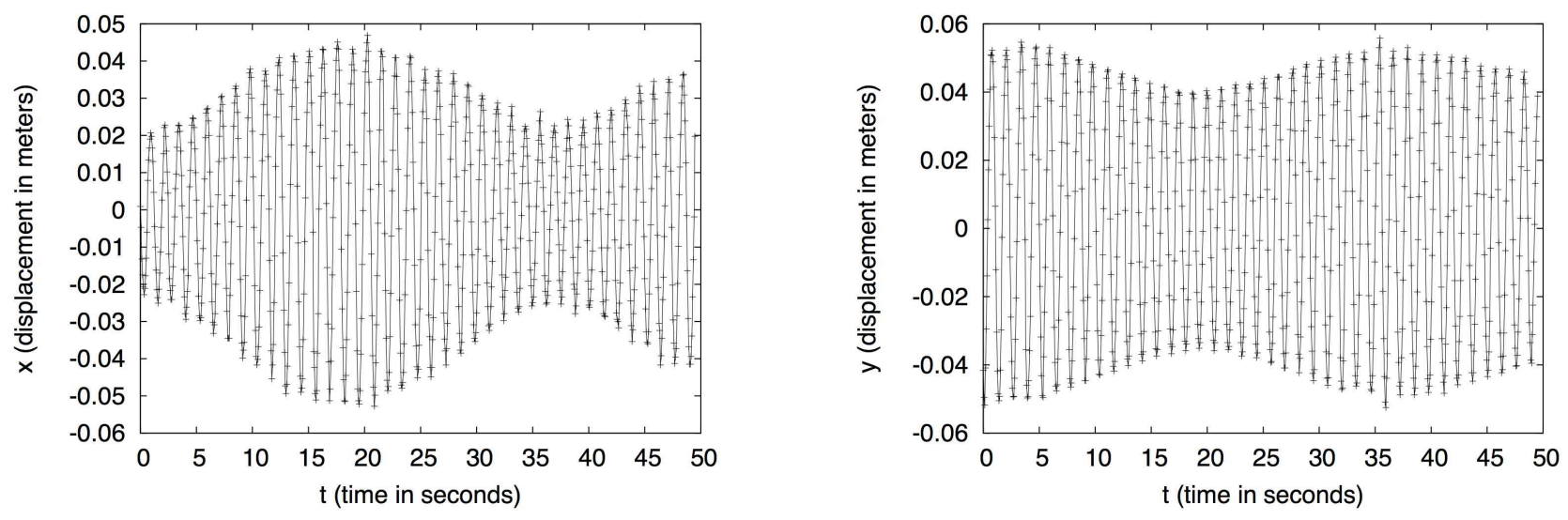

FIG. 8: Experimental data showing the motion of the pendula when the magnets are turned on weakly, so that there is a weak loss and gain, and the pendula are strongly coupled (the tension in the supporting rope is lowered to 400 grams). Observe that the Rabi power oscillations in Fig. 7 persist. This means that the system is in a region of unbroken $\mathcal{P} \mathcal{T}$ symmetry. 

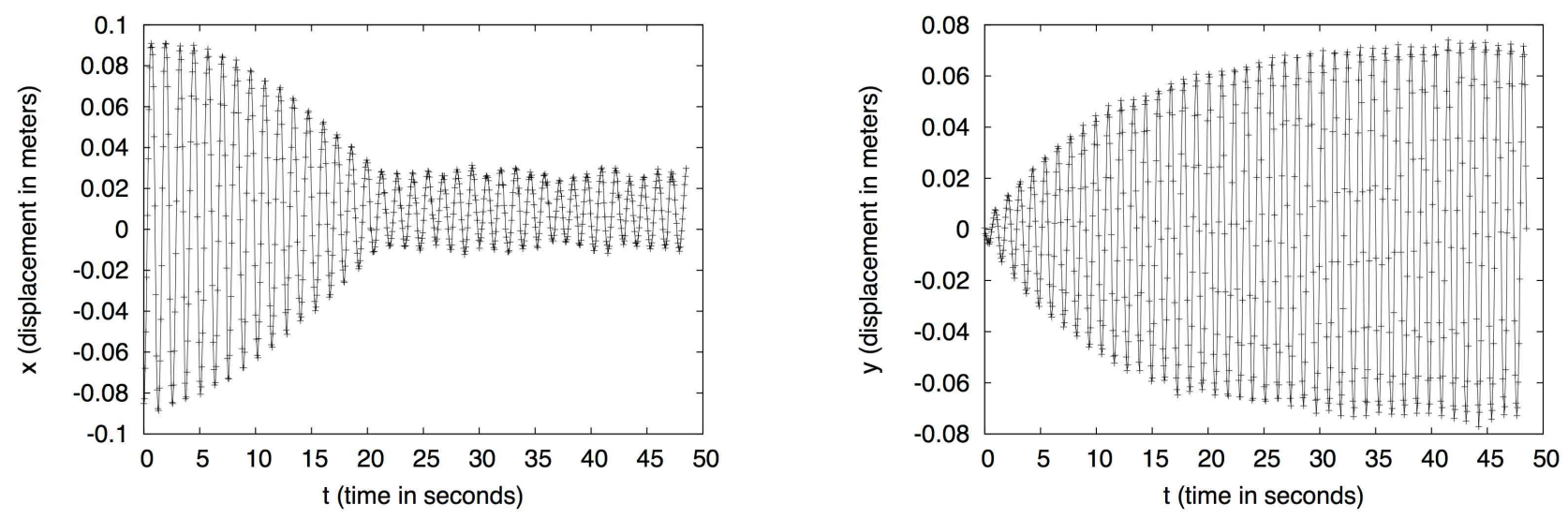

FIG. 9: Experimental data showing the motion of the pendula when the magnets are turned on strongly and the coupling of the pendula is weak (the tension in the supporting string is raised to 600 grams). Observe the the Rabi oscillations have ceased. This is the signal that the system is in a region of broken $\mathcal{P} \mathcal{T}$ symmetry.

[1] C. M. Bender and S. Boettcher, Phys. Rev. Lett. 80, 5243 (1998); P. Dorey, C. Dunning, and R. Tateo, J. Phys. A 34, L391 (2001) and ibid. 34, 5679 (2001).

[2] C. M. Bender, D. C. Brody, and H. F. Jones, Phys. Rev. Lett. 89, 270401 (2002).

[3] C. M. Bender, D. C. Brody, and H. F. Jones, Am. J. Phys. 71, 1095 (2003).

[4] C. Zheng, L. Hao, and G. L. Long, arXiv:1105.6157

[5] J. Rubinstein, P. Sternberg, and Q. Ma, Phys. Rev. Lett. 99, 167003 (2007).

[6] A. Guo, G. J. Salamo, D. Duchesne, R. Morandotti, M. Volatier-Ravat, V. Aimez, G. A. Siviloglou, and D. N. Christodoulides, Phys. Rev. Lett. 103, 093902 (2009).

[7] C. E. Rüter, K. G. Makris, R. El-Ganainy, D. N. Christodoulides, M. Segev, and D. Kip, Nat. Phys. 6, 192-195 (2010).

[8] K. F. Zhao, M. Schaden, and Z. Wu, Phys. Rev. A 81, 042903 (2010).

[9] Z. Lin, H. Ramezani, T. Eichelkraut, T. Kottos, H. Cao, and D. N. Christodoulides, Phys. Rev. Lett. 106, 213901 (2011).

[10] L. Feng, M. Ayache, J. Huang, Y.-L. Xu, M.-H. Lu, Y.F. Chen, Y. Fainman, and A. Scherer, Science 333, 729 (2011).
[11] J. Schindler, A. Li, M. C. Zheng, F. M. Ellis, T. Kottos, Phys. Rev. A 84, 040101(R) (2011).

[12] S. Bittner, B. Dietz, U. Günther, H. L. Harney, M. MiskiOglu, A. Richter, and F. Schäfer, Phys. Rev. Lett. 108, 024101 (2012).

[13] J. L. Rosner and S. A. Slezak, Am. J. Phys. 326, 2717 (2011).

[14] K. R. Schubert and J. Stiewe, J. Phys. G: Nucl. Part. Phys. 39, 033101 (2012).

[15] A. Reiser, K. R. Schubert, and J. Stiewe, arXiv: 1203.4703.

[16] P. Dorey, C. Dunning, and R. Tateo, J. Phys. A: Math. Gen. 34, 34, 5679 (2001).

[17] P. Dorey, C. Dunning, and R. Tateo, J. Phys. A: Math. Gen. 40, R205 (2007).

[18] Details of the video camera system: Single user VisiLog, full version with advanced USB video camera and gooseneck stand, supplied by 2012 ScienceScope, Writhlington School, Knobsbury Lane, Writhlington, Radstock, BA3 3NQ, UK.

[19] K. Makris, R. El-Ganainy, D. N. Christodoulides, and Z. H. Musslimani, Phys. Rev. Lett. 100, 103904 (2008). 